IMECE2009-12762

\title{
A MICROMACHINED EARTH SENSOR BASED ON MEASURING THE GRAVITY GRADIENT TORQUE
}

\author{
K. Ghose \\ Microsystems for Space Technologies Laboratory, \\ Ecole Polytechnique Fédérale de Lausanne \\ Neuchâtel, Switzerland
}

\begin{abstract}
We present the fabrication and testing of a novel MEMS inertial sensor that directly measures the gravity gradient in low Earth orbit in order to sense the relative orientation of a satellite with respect to the Earth. Instead of the current Earth sensing methods that determine the Earth vector by sensing the Earth's IR emission, we present a much lighter and more compact MEMS-based approach that determine the Earth vector by measuring the Gravity Gradient Torque on an elongated silicon proof mass. Current Earth sensors require optical access on multiple faces of the satellite. This MEMS-based approach does not require optical access.
\end{abstract}

\section{EARTH SENSOR DESIGN}

Due to the inverse square scaling of the gravitational force with distance, different segments of an elongated object in Earth orbit at slightly different distances from the Earth's center are subject to minute differences in gravitational force. This results in a net torque, called the gravity gradient torque (GGT) around the center of mass of the elongated body, whose magnitude is dependent on the angle of orientation of the elongated body to the Earth normal [1].

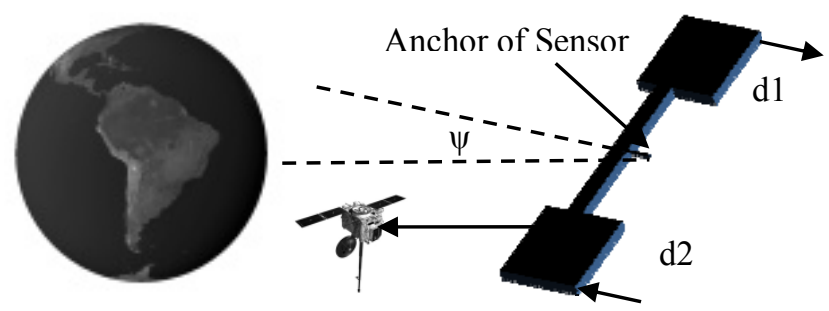

Fig. 1. The MEMS based Earth sensor on board a satellite. Its displacement (d1, d2) depends on the angle $\psi$ of its axis of minimum moment of inertia to the Earth normal

\author{
H.R. Shea \\ Microsystems for Space Technologies Laboratory, \\ Ecole Polytechnique Fédérale de Lausanne \\ Neuchâtel, Switzerland
}

The equation for GGT for a body in orbit around the Earth is given as [1]

$$
G G T=3 \mu \cdot I \cdot \sin (2 \varphi) / 2 R^{3}
$$

$\mu$ is the gravity constant of the Earth, I is the maximum moment of inertia of the elongated body, $\psi$ is the angle between the axis of minimum moment of inertia of the body and the vector to the Earth center, and $\mathrm{R}$ is the radius of the orbit.

A suitable inertial sensor to detect torque is a pendulum consisting of an elongated mass suspended from a compliant spring. This pendulum is placed on board a satellite with the spring being the only link between the mass and the satellite body as in fig. 1. The orientation of the satellite determines the orientation of the spring, and hence approximately of the pendulum mass with respect to the Earth normal. From Equation (1), when the satellite is oriented such that the angle $\psi$ is 0 there is no GGT on the pendulum. When the satellite changes its orientation the angle $\psi$ changes and the mass experiences GGT according to Equation (1). When subjected to torque the mass rotates slightly (fraction of a degree) and the spring from which it is suspended bends. The bending of the spring provides an opposite torque and the mass reaches a steady state displacement whose magnitude is dependent on the angle $\psi$ and on the device geometry. This displacement is relative to the satellite body. At the ends of the pendulum mass the displacement due to the rotation can be measured to determine $\psi$ and therefore the external torque.

For our geometry, the maximum GGT on the pendulum is of the order $10^{-13} \mathrm{~N} . \mathrm{m}$. This necessitates the use of a very soft spring to obtain a sufficient displacement from this minute torque. To obtain a good signal to noise ratio, the mass, and therefore moment of inertia of the pendulum mass can be increased, thereby obtaining a larger GGT [2]. While this presents no problem in microgravity, a larger mass results in a 
device that is more prone to breakage on Earth, and also limits the number of devices that can be fabricated per wafer. At the same time, the compliance of the spring can be increased, thus providing greater displacement for a given torque. Even with a highly compliant spring, the displacements that are obtained in microgravity in a low Earth orbit of $300 \mathrm{~km}$ are in the order of nanometers. The limitation on the spring softness is imposed by the need to be able to test the device in a one gravity environment on Earth. The spring dimensions are chosen to provide high compliance to in-plane torque, but very high stiffness to gravity perpendicular to the chip plane to be able to test it on Earth.

We use a $1 \times 4 \mathrm{~cm}^{2}$ area, $600 \mu \mathrm{m}$ thick silicon pendulum suspended by a $1 \mathrm{~mm}$ long, $15 \mu \mathrm{m}$ wide, $100 \mu \mathrm{m}$ deep spring. Since the GGT depends on the moment of inertia, the shape of the mass was chosen so that its moment of inertia is maximized. Due to the $10^{-13} \mathrm{Nm}$ GGT on the mass in microgravity, the expected displacement of the extreme edge of the pendulum on the $\mathrm{X}$ axis, in the direction of the positive $\mathrm{Z}$ axis is in the $\mathrm{nm}$ range, as indicated in fig. 3. The barbell shape also makes it easier to implement a differential readout.

The maximum moment of inertia of the barbell is $6.95 \times$ $10^{-8} \mathrm{~kg} \cdot \mathrm{m}^{2}$ and the spring constant is $5.0625 \times 10^{-6}$. The fundamental mode of the pendulum is $1.36 \mathrm{~Hz}$, from analytical calculation and verified by simulation in ANSYS. This mode corresponds to the swing of the pendulum about the Y axis. It should be noted that this frequency is outside the typical vibration spectrum onboard an operating satellite.

To detect this nanometer level motion we use an integrated fiber based interferometer due to high sensitivity of interferometric methods [3]. Though its sensitivity is lower than that of devices that detect displacement by electron tunneling, it is far more robust and does not require special handling.

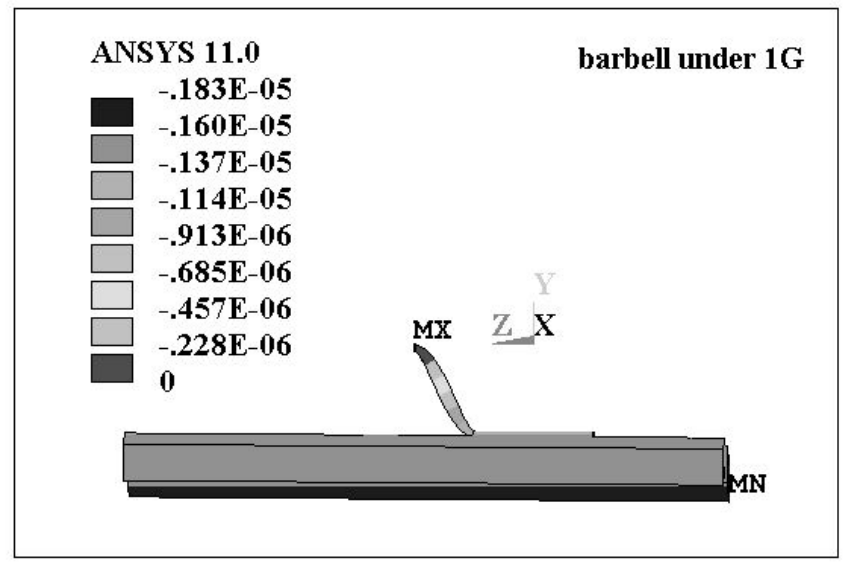

Fig. 2. FEM simulation showing the side view of the barbell mass suspended for testing in one gravity. The $1 \times 4 \mathrm{~cm}^{2}$ area, $600 \mu \mathrm{m}$ thick, $0.34 \mathrm{~g} \mathrm{Si}$ mass is level to the ground and sags by only $1.6 \mu \mathrm{m}$. ANSYS increases the scale to clearly show the displacement of the device under simulation.

To be able to test the device, it should be freely suspended. The shape of the barbell is such that its center of mass is at the center of the spring. This prevents the mass from sagging out of plane, as seen in fig. 2 and allows it to be levelly suspended for testing.

This mass distribution also allows the Earth sensor to operate even when the satellite is spinning. Fig. 4 shows the FEM simulation of the displacement along the $\mathrm{Z}$ axis when the Earth sensor is on a spinning satellite. The displacement along the $\mathrm{Z}$ axis due to GGT is measured in order to determine the Earth vector. When the pendulum is spinning at $0.02 \mathrm{rad} / \mathrm{sec}$ $(\sim 1.15 \% / \mathrm{sec})$ in the XZ plane, around a center of rotation located at $1 \mathrm{~m}$ from the center of mass at an angle of $45^{\circ}$ to the positive $\mathrm{X}$ axis, the pendulum displacement along the positive $\mathrm{Z}$ axis is only $0.04 \mathrm{~nm}$, much less than the displacement due to the GGT. If the Earth sensor is placed closer to the center of spin of a satellite the displacement will scale linearly with the distance from the center.

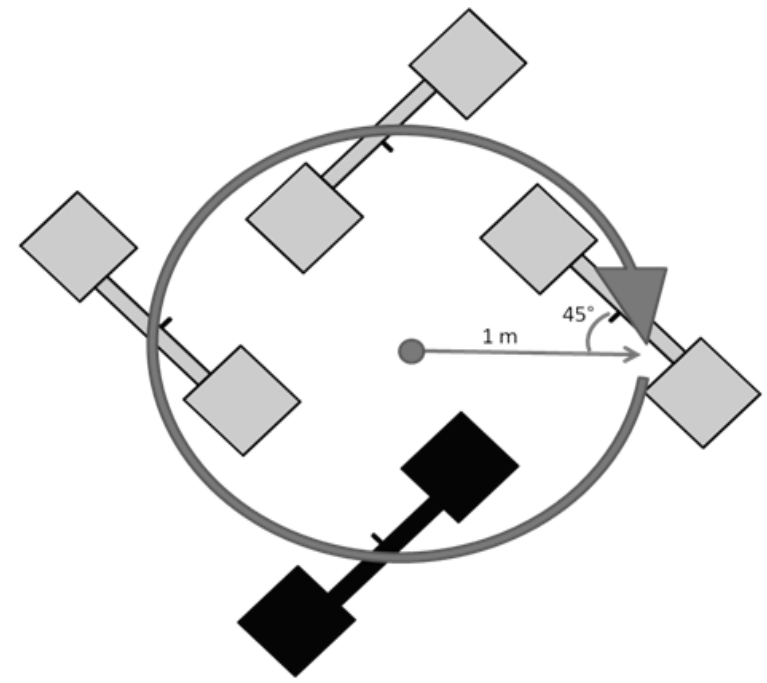

Fig. 3. Schematic of the worst case orientation of a Earth sensor chip on a spinning satellite. The simulated displacement of the sensor due to this rotation is shown in fig. 4

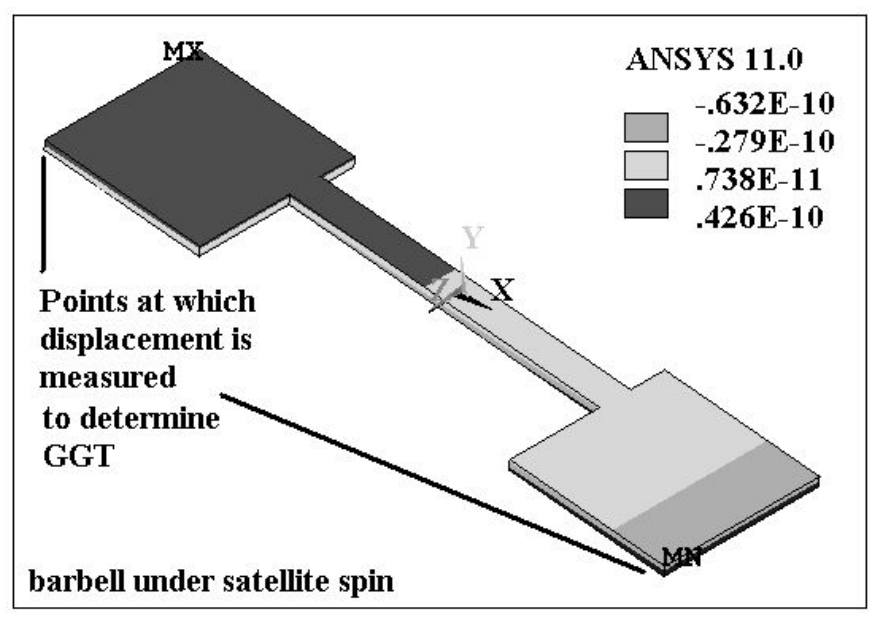

Fig. 4. FEM simulation of pendulum when it is on a spinning satellite with an angular velocity of $0.02 \mathrm{rad} / \mathrm{sec}$ in the $\mathrm{XZ}$ plane, with the sensor oriented as shown in fig. 3 . The legend indicates displacement in meters 


\section{FABRICATION OF THE EARTH SENSOR}

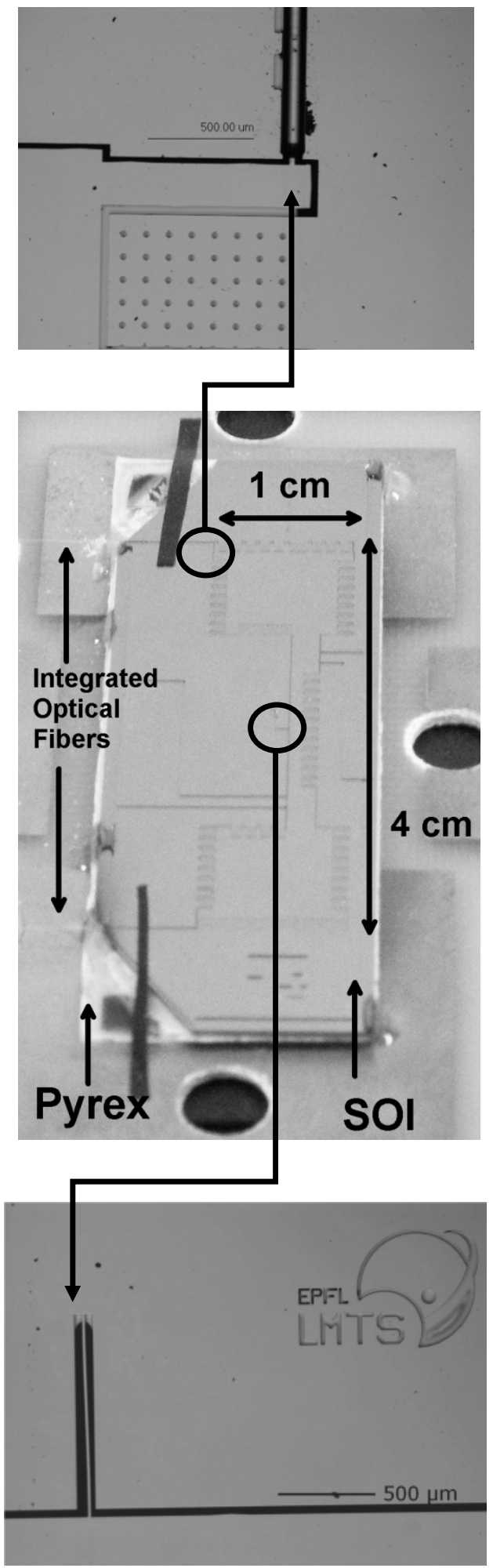

Fig. 5: Top: Optical Micrograph of the fiber based interferometer integrated onto the Earth sensor chip; Middle: Photograph of the fabricated Earth sensor mounted on a PCB for test. The outline of the barbell shaped pendulum can be seen; Top: detail of mounted optical fiber.- Bottom: Optical micrograph of the released spring.
Though the sensor is designed for microgravity it is fabricated and tested in $1 \mathrm{G}$, so it must be robust enough to survive handling on Earth. The major fabrication challenge was to develop a process flow that limits the motion of the $1 \times 4 \mathrm{~cm}^{2}$ long proof mass to a few microns on all 3 axes, to make the device robust enough for testing on Earth, while choosing a spring mass combination sensitive enough to measure the gravity gradient torque. The pendulum has a mass of $0.34 \mathrm{~g}$. Both device and handle layers of an SOI wafer $(500 \mu \mathrm{m}$ handle, $1 \mu \mathrm{m}$ oxide, $100 \mu \mathrm{m}$ device layers) are etched (DRIE) to form the mass of the pendulum, while the spring is fabricated only from the device layer.

The motion limitation is built into the fabrication process, so that the mass is protected before final release. The device layer has stoppers etched into it which restrict the motion of the sensor mass in the X-Y plane. Motion in the positive $Z$ direction is restricted by having part of the device layer overhang the handle layer of the pendulum mass. To restrict motion in the $\mathrm{Z}$ plane, and completely constrain the mass before final release, a two-step release process is followed. The chip is first released from the wafer using HF vapor. Then a Pyrex chip with spikes giving $30 \mu \mathrm{m}$ of clearance to the bottom of the handle layer of the mass is bonded to the handle layer as in [4]. Then the SOI-Pyrex chip is again put into HF vapor to release the proof mass from the chip frame. The device survives routine handling in the laboratory with no special precautions needed to prevent the proof mass from moving.

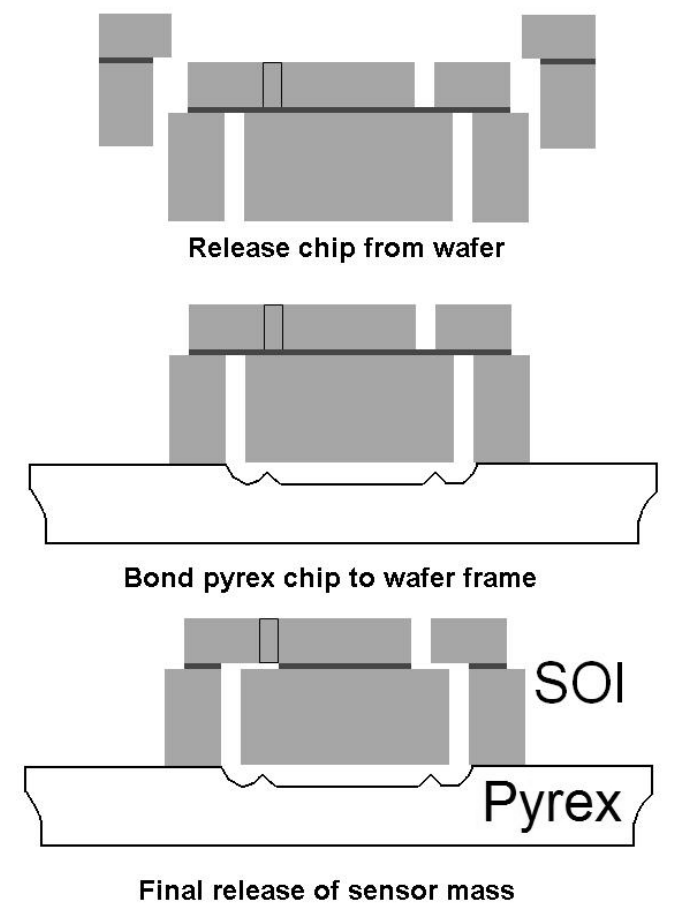

Fig. 6: Schematic of the two-step release process. To prevent the spring from breaking during fabrication, the proof mass is only released in HF vapor after the SOI chip is bonded to an etched Pyrex wafer. 


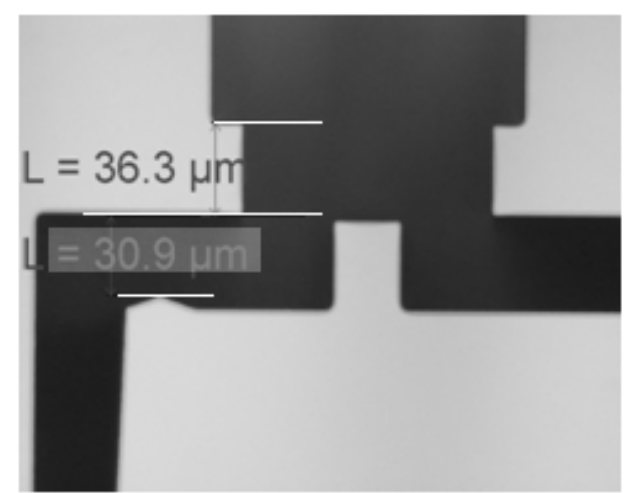

Fig. 7: Optical micrograph of fiber mounting area of chip; Inplane stoppers that restrict proof mass motion to 30 microns, and in this image prevent it from hitting the fiber tip

Fig. 5 shows the fabricated and released Earth sensor. The integrated fibers that are used to detect the displacement of the mass can be seen on the left side of the middle of fig. 5. To build the interferometer, we etch a groove into the device layer of the SOI wafer during DRIE with clamps to hold the fiber in the groove. The fiber can be positioned at a known distance from the sensor mass as seen in figs. 5 and 7.

\section{TESTING OF THE EARTH SENSOR}

To replicate the $\mathrm{nm}$ scale motion expected from GGT in microgravity to test the chip on Earth, we mount the device on a piezo driven tilt stage. As the stage moves through very small angles, the sensor mass moves by $\mathrm{nm}$ relative to the fiber tip. While tilting the piezo stage, the tilt angle was monitored using an external laser reflecting off the SOI chip onto a Position Sensitive Detector (PSD). By recording the distance the laser spot moves on the PSD, the angle through which the piezo tilts can be determined.

The tilt due to the piezo stage was from $0-0.014^{\circ}$ and back to $0^{\circ}$. From FEM simulations, the displacement of the sensor mass relative to the fiber tip was determined to be $0-21$ $\mathrm{nm}$ for this tilt. The resulting photocurrent at the measuring photodiode of the fiber interferometer is recorded as seen in fig. 8 , showing the mass is freely suspended, and that the interferometric readout is capable of detecting motion over a few nanometers.

\section{CONCLUSION}

We designed and fabricated a micromachined silicon pendulum and suspension to measure the gravity gradient by measuring the torque on the pendulum due to the gravity gradient. The design of the pendulum maximizes its moment of inertia and the suspension is designed to maximize the displacement for a given GGT, while minimizing the displacement due to gravity on Earth (so as to allow easy testing on Earth). The displacement of this pendulum due to satellite spin is negligible, allowing the Earth sensor to operate regardless of the satellite spinning or tumbling. A significant part of the work involved successfully fabricating the sensor. It survives routine handling in the lab, which indicates a survivability of at least tens of gravities. The expected displacements in micro-gravity were duplicated successfully on Earth which allowed us to test the readout scheme, based on an optical fiber based interferometer. The current measurements indicate that the interferometer can measure displacements with an accuracy of around $1 \mathrm{~nm}$. This corresponds to an uncertainty of $\sim 25^{\circ}$. These measurements were done by direct measurement of the photocurrent. Using phase sensitive detection, and closed loop control we should be able to obtain much better sensitivity, ultimately allowing the MEMS Earth sensor to achieve a target resolution in the Earth pointing vector of at least $5^{\circ}$.

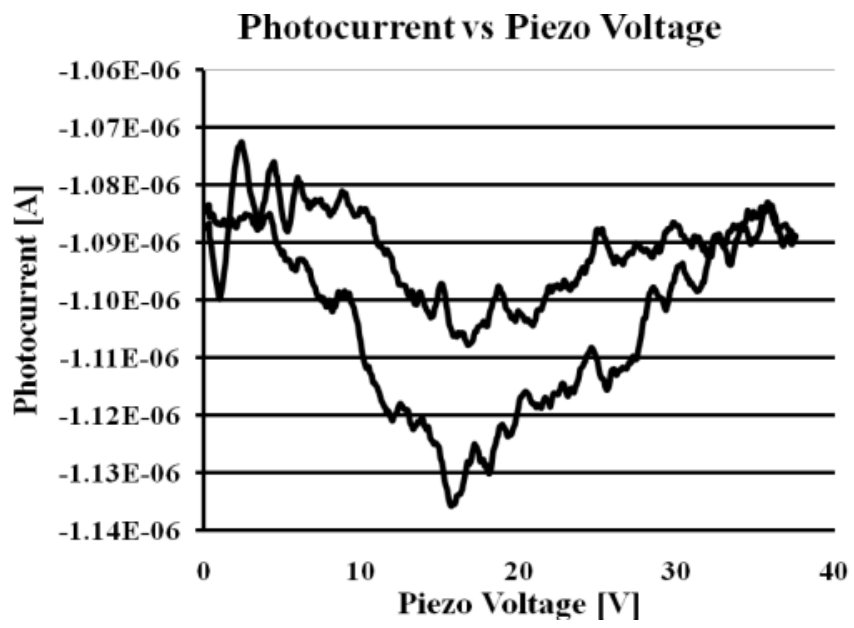

Fig. 8: Photodiode current vs. voltage applied to the tip-tilt stage piezo (up and down). The corresponding maximum displacement of the mass is $21 \mathrm{~nm}$. Some hysteresis is seen since the piezo is operated in open loop mode. This data shows the mass is moving as the device is tilted and the motion can be sensed with an accuracy of $1 \mathrm{~nm}$.

\section{ACKNOWLEDGMENTS}

This work is partly funded by the European Space Agency.

\section{REFERENCES}

[1] J.R. Wertz, W.J. Larson, 1999, Space Mission Analysis and Design, Kluwer Academic Publishers, Dordrecht

[2] T.B. Gabrielson, 1999, "Mechanical-Thermal Noise in Micromachined Acoustic and Vibration Sensors", IEEE Transactions on Electron Devices, 40, pp. 903-909

[3] D. Rugar, H.J. Mamin, P. Guethner, 1989, "Improved fiber-optic interferometer for atomic force spectroscopy", Appl. Phys. Lett., 55, pp. 2588-2590

[4] T.P. Swiler, U. Krishnamoorthy, P.J. Lewis, M.S. Baker, D.M. Tanner, 2008, "Challenges of designing and processing extreme low-G microelectromechanical system (MEMS) accelerometers", Reliability, Packaging, Testing, and Characterization of MEMS/MOEMS VII Conference, San Jose, 688400 